\title{
The formation of homogentisate in the biosynthesis of tocopherol and plastoquinone in spinach chloroplasts
}

\author{
Erich Fiedler, Jürgen Soll, and Gernot Schultz \\ Institut für Tierernährung, Arbeitsgruppe für Phytochemie, Tierärztliche Hochschule, \\ Bischofsholer Damm 15, D-3000 Hannover 1, Federal Republic of Germany
}

\begin{abstract}
Homogentisate is the precursor in the biosynthesis of $\alpha$-tocopherol and plastoquinone-9 in chloroplasts. It is formed of 4-hydroxyphenylpyruvate of the shikimate pathway by the 4-hydroxyphenylpyruvate dioxygenase. In experiments with spinach the dioxygenase was shown to be localized predominatedly in the chloroplasts. Envelope membranes exhibit the highest specific activity, however, because of the high stromal portion of chloroplasts, $60-80 \%$ of the total activity is housed in the stroma. The incorporation of 4-hydroxyphenylpyruvate into 2-methyl-6-phytylquinol as the first intermediate in the tocopherol synthesis by the two-step-reaction: 4-Hydroxyphenylpyruvate $\rightarrow$ Homogentisate $\stackrel{\text { Phytyl-PP }}{\longrightarrow}$ 2-Methyl-6-phytylquinol was demonstrated by using envelope membranes. Homogentisate originates directly from 4-hydroxyphenylpyruvate of the shikimate pathway. Additionally, a bypass exists in chloroplasts which forms 4-hydroxyphenylpyruvate from tyrosine by an L-amino-acid oxidase of the thylakoids and in peroxisomes by a transaminase reaction. Former results about the dioxygenase in peroxisomes were verified.
\end{abstract}

Key words: Chloroplast - Homogentisate $-4-\mathrm{Hy}$ droxyphenylpyruvate dioxygenase - Plastoquinone - Spinacia - Tocopherol.

\section{Introduction}

Homogentisate is well known as the aromatic precursor in the biosynthesis of $\alpha$-tocopherol and plastoquinone in the chloroplast (Whistance and Threlfall 1970; Hutson and Threlfall 1980; Goodwin 1965). The envelope membranes were found to catalyze prenylation to the corresponding meth- ylprenylquinol and also the subsequent reaction steps (Soll et al. 1980) (Fig. 1). The homogentisate synthesis from 4-hydroxyphenylpyruvate has not been elucidated as yet though the shikimate pathway is active in spinach chloroplasts (Bickel et al. 1978; Bickel and Schultz 1979; Buchholz and Schultz 1980).

Considerable formation of homogentisate from both 4-hydroxyphenylpyruvate and tyrosine had only been described in peroxisomes (Bickel et al. 1979). The occurrence of a homogentisateforming system in thylakoids of Lemna gibba was reported (Löffelhardt and Kindl 1979). The present paper indicates that the stroma of spinach chloroplasts is the main site of the 4-hydroxyphenylpyruvate dioxygenase.

\section{Materials and methods}

Radiochemicals. L-[ring-3,5- $\left.{ }^{3} \mathrm{H}\right]$ tyrosine $\left(1889 \mathrm{GBq}^{\mathrm{mmol}}{ }^{-1}\right)$ was purchased from NEN (Dreieich, FRG). L-[ring-3,5- $\left.{ }^{3} \mathrm{H}\right]-4-$ hydroxyphenylpyruvate was enzymically prepared according to Lindblad (1971), modified as follows: $18,8 \mathrm{MBq}$ L-[ring-3,5${ }^{3} \mathrm{H}$ ] tyrosine, 16,7 nkat L-amino acid oxidase (Sigma Munich, FRG), crude dried venom from Crotalus adamanteus), and $16,7 \mu \mathrm{kat}$ catalase (Sigma purified powder from bovine liver) were incubated for $30 \mathrm{~min}$ at room temperature in a total volume of $200 \mu 150 \mathrm{mM}$ sodium phosphatebuffer, $\mathrm{pH}$ 6.5. After addition of $50 \mu \mathrm{g}$ unlabeled 4-hydroxyphenylpyruvate, the mixture was layered on a $3.10 \mathrm{~mm}$ column Dowex $50 \mathrm{~W} \times 8$, 200-400 mesh (Serva, Heidelberg, FRG) equilibrated with $0.1 \mathrm{M} \mathrm{HCl}$. The column was eluted with ice cold water. The first $100 \mu \mathrm{l}$ eluate were discharged; the next $2 \mathrm{ml}$ were collected, acidified ( $\mathrm{pH}$ 2), and extracted four times with ice-cold diethylether. Then, 50-200 $\mu 1 \mathrm{H}_{2} \mathrm{O}$ were added and the ether removed. The radiochemical purity was $>93 \%$ (Fig. 2). The yield of labeled product was ca $60 \%$. 4-Hydroxyphenylpyruvate is unstable and degrades to a small extent to 4-hydroxybenzaldehyde and 4-hydroxyphenyl acetic acid during the chromatography. Control experiments did not show tyrosine in the extract containing 4-hydroxyphenylpyruvate. Due to its low stability, 4- 


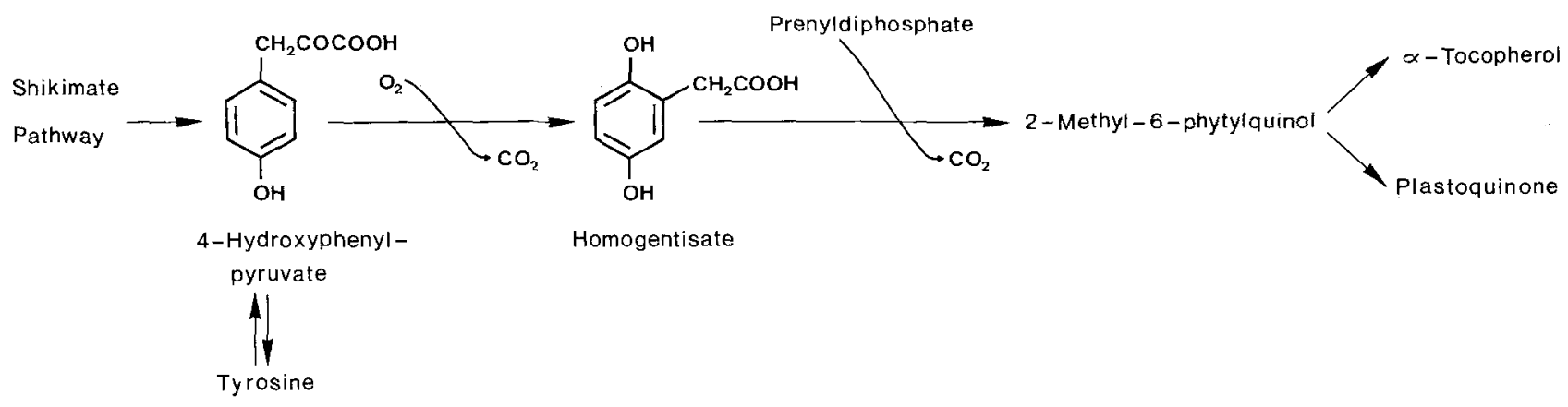

Fig. 1. The role of 4-hydroxyphenylpyruvate and homogentisate in biosynthesis of the prenylquinones $\alpha$-tocopherol and plastoquinone in chloroplasts

hydroxyphenylpyruvate had always to be prepared immediately before use. The specific radioactivity was $66 \mathrm{GBq} \mathrm{mmol}{ }^{-1}$.

Isolation of purified chloroplasts. Intact chloroplasts were prepared from spinach leaves according to Nakatani and Barber (1977). They were further purified by centrifugation through gradients of $90 \%$ Percoll, essentially according to Haas et al. (1980). To assure free passage of tyrosine and 4-hydroxyphenylpyruvate across the envelope, the purified chloroplasts were osmotically shocked in hypotonic buffer solution $(10 \mathrm{mM} 4-(2$ hydroxyethyl)-1-piperazine ethane sulfonic acid (HEPES), $\mathrm{pH} 7.6 ; 4 \mathrm{mM} \mathrm{MgCl}$ ).

Isolation of chloroplast subfractions. Purified intact chloroplasts were ruptured by hypotonic lysis. Envelope membranes, thylakoids, and membrane-free stroma were obtained by centrifugation on a discontinuous sucrose gradient (Douce and Joyard 1979). Thylakoids were washed twice prior to use.

Isolation of peroxisomes. Leaf peroxisomes from spinach were isolated as described in Buchholz et al. (1979) and were assayed for NADH-hydroxypyruvate reductase (EC 1.1.1.29) as a marker enzyme for peroxisomes (Tolbert et al. 1970).

Reaction mixture. If not otherwise defined, the complete reaction mixture contained: $50 \mathrm{mM}$ HEPES, $\mathrm{pH} 7.6 ; 4 \mathrm{mM} \mathrm{MgCl}_{2}$; $2 \mathrm{mM} \mathrm{MnCl}_{2} ; 4 \mathrm{mM}$ sodium-ascorbate; L-[ring-3,5- $\left.{ }^{3} \mathrm{H}\right]$ tyrosine or L-[ring-3,5- $\left.{ }^{3} \mathrm{H}\right]-4$-hydroxyphenylpyruvate (for further details see figures and text). The assays were incubated in the dark at $20^{\circ} \mathrm{C}$ for $30-50 \mathrm{~min}$. The final volume of $1 \mathrm{ml}$ contained not less than $2 \mathrm{mg}$ chlorophyll in experiments with broken chloroplasts or not less than $1 \mathrm{mg}$ chloroplast subfraction protein or peroxisomal protein, respectively. Protein content was determined according to Lowry et al. (1951) and chlorophyll according to Arnon (1949).

Purification and identification of labeled products. Aliquots $(200 \mu 1)$ were taken at different times. The reaction was stopped with $750 \mu$ l of a $2: 1$ mixture of methanol/chloroform to obtain a monophasic solution. Carrier substance $(700 \mu \mathrm{g}$ per aliquot), identical to the expected labeled product, was added. The aqueous phase was removed after $500 \mu 1 \mathrm{H}_{2} \mathrm{O}$ had been added and the mixture had been thoroughly shaken. Residual labeled 4-hydroxyphenylpyruvate was converted to the stable oxime by $\mathrm{NH}_{2} \mathrm{OH}(10-30 \mu \mathrm{mol})$ in a volume of $2 \mathrm{ml}$ (Löffelhardt and Kindl 1979). After 20 min at room temperature, the mixture was acidified and extracted four times with diethylether. Homogentisate is susceptible to oxidation, so it has to be immediately purified. It was chromatographed on silicagel/kieselguhr layers (Merck, Darmstadt, FRG) with benzene/methanol/acetic acid

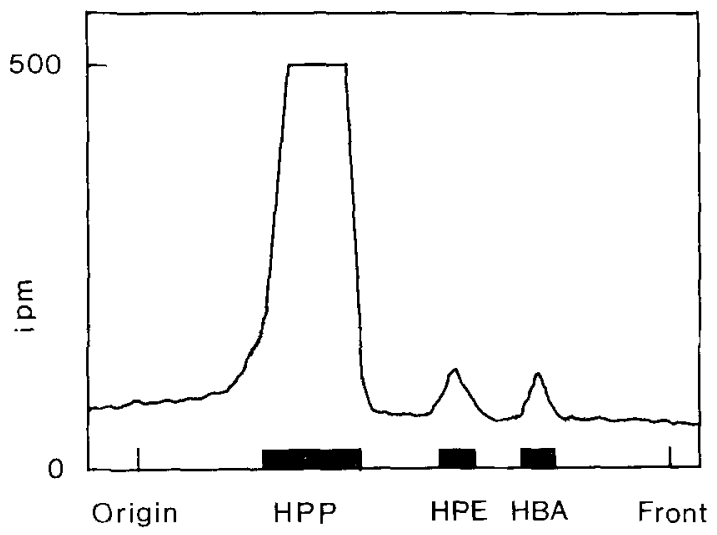

Fig. 2. Radiochemical purity of 4-hydroxyphenylpyruvate. Scan after thin layer chromatography of 4-hydroxyphenylpyruvate on silicagel (Schleicher \& Schüll G 1500, Dassel, FRG) with benzene/methanol/acetic acid $45: 8: 4$ as solvent system. 4-hydroxyphenylpyruvate (HPP); 4-hydroxyphenyl acetic acid (HPA); 4-hydroxybenzaldehyde (HBA)

$45: 8: 4$. Its spot at $R_{F} 0.35$ was eluted with dry diethylether. Defined oxidation with $\mathrm{FeCl}_{3}$ yields p-benzoquinone acetic acid (Whistance and Threlfall 1970) which is much more stable. After chromatography on silicagel/kieselguhr layers with dichlormethane/toluene/formic acid 5/4/1 as solvent system the radioactive areas at $R_{F} 0.4$ were subjected to liquid scintillation counting. All substances were cochromatographed with authentic samples and detected by ultraviolet quench at $254 \mathrm{~nm}$. The structure of $\mathrm{p}$-benzoquinone acetic acid was verified by nuclear magnetic resonance (NMR)- and mass spectrometry.

\section{Results}

Formation of homogentisate from 4-hydroxyphenylpyruvate in peroxisomes and chloroplasts. Experiments in our laboratory (Fiedler unpublished data) have shown that the formation of homogentisate is independent from light. Ascorbate is essential, the optimal range is $2-5 \mathrm{mM}$. Peroxisomes have their own 4-hydroxyphenylpyruvate dioxygenase activity at 444 pkat homogentisate per kg peroxisomal protein, which was 10 -fold higher than that 
Table 1. Correlation between the distribution of 4-hydroxyphenylpyruvate dioxygenase and markers of the organelles. Chloroplasts and peroxisomes were isolated from the same charge of spinach (for details see text and Materials and methods)

Chlorophyll g per $\mathrm{kg}$ protein

$$
\begin{array}{ll}
\text { NADH-hydroxypyruvate- } & \begin{array}{l}
\text { 4-hydroxyphenyl- } \\
\text { reductase }
\end{array} \\
\text { pyruvate dioxygenase }
\end{array}
$$

Specific activity in pkat per $\mathrm{kg}$ protein
Ratio of specific activities of 4-hydroxyphenylpyrivate dioxygenase

NADH-hydroxypyruvate reductase of chloroplasts, using $10 \mu \mathrm{M}$ 4-hydroxyphenylpyruvate. Chloroplasts were tested for NADH-hydroxypyruvate reductase as a marker enzyme for peroxisomes (Tolbert et al. 1970). The ratio of the activity of the dioxygenase to the NADH-hydroxypyruvate reductase is given in Table 1 . The contamination found by peroxisomes (expressed in terms of activity of NADH-hydroxypyruvate reductase) was far too low to explain the dioxygenase activity present in chloroplasts. The ratio of plastidic protein to peroxisomal protein in leaves is greater than 50:1 (Tolbert 1971; Beevers 1971). Hence, the chloroplasts play a predominant role in the homogentisate synthesis. More than $80 \%$ of the total homogentisate formation takes place in these organelles.

Formation of homogentisate from tyrosine in peroxisomes and chloroplasts. 4-hydroxyphenylpyruvate can be formed from tyrosine by a transaminase and/or L-amino acid oxidase reaction. Peroxisomes were incubated with tyrosine $(3.2 \mu \mathrm{M})$ and $1 \mathrm{mM} \alpha$-ketoglutarate plus $2 \mathrm{mM}$ pyridoxal phosphate to test the transaminase. This resulted in a 12 -fold increase of the conversion rate from 9,4 to 112 pkat homogentisate (formed via 4-hydroxyphenylpyruvate) per $\mathrm{kg}$ peroxisomal protein. In chloroplasts 4-hydroxyphenylpyruvate formation is not stimulated by adding $\alpha$-ketoglutarate (Bickel et al. 1979). Its synthesis is catalyzed by an L-amino acid oxidase.

Localisation of L-amino acid oxidase in chloroplasts. Evidence was found for a thylakoid-bound L-amino acid oxidase (3.6 pkat homogentisate per $\mathrm{kg}$ thylakoid protein (from tyrosine $(1.85 \mu \mathrm{M})$ in a coupled reaction of oxidase plus dioxygenase). This enzyme could neither be demonstrated in the stroma nor in the envelope (data not shown). A thylakoid-bound L-amino acid oxidase was also found in Anacystis nidulans (Löffelhardt 1977).

Specific activity of chloroplast subfractions in homogentisate formation. 4-Hydroxyphenylpyruvate

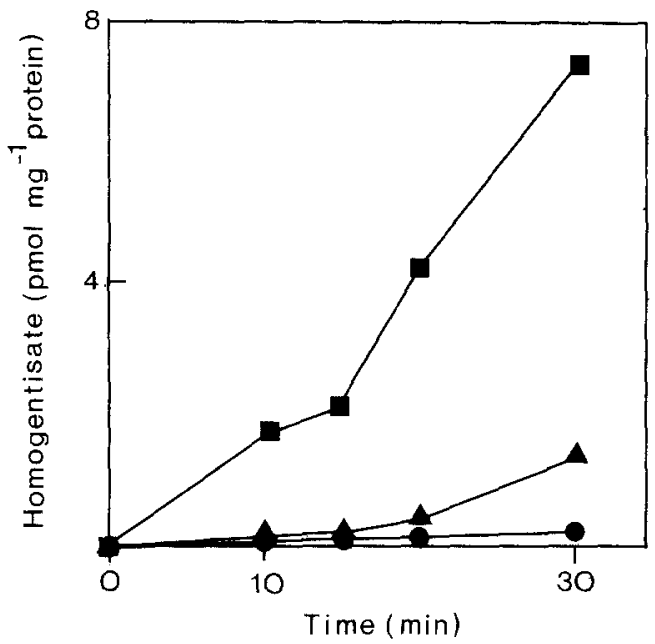

Fig. 3. Formation of homogentisate from 4-hydroxyphenylpyruvate $(8 \mu \mathrm{M})$ by chloroplast subfractions. Envelope membranes ( $\bullet$ ); stroma phase (4); thylakoids $(\bullet)$. For further details see Materials and methods

dioxygenase activity was shown in all three compartments, stroma, thylakoids, and envelope. When stromal and membrane fractions are combined, the rate of homogentisate formation is not stimulated (data not shown). Envelope membranes exhibit the highest specific activity (Fig. 3 and Table 2). Because protein ratios in stroma, thylakoids, and envelopes are about 50:50:1, the chloroplast capacity of homogentisate synthesis is localized to $60-80 \%$ in stroma, but the homogentisate formation of thylakoids and envelope membranes is not negligible. Both membranes possess their own activity. This activity cannot be attributed to a contamination by stroma. For criteria of purity of subfractions see Douce and Joyard (1977); Douce et al. 1973; Douce and Joyard (1979). No ribolose-1,5-bis-phosphate carboxylase activity could be detected in the envelope fraction (assay according to McNeil et al. 1981; data not shown). A contamination of thylakoids by envelope was not likely, because the homogentisate-phytyldiphosphate-transferase localized only in the envelope (Soll et al. 1980) was not detectable. 
Table 2. Formation of homogentisate in dependence on different 4-hydroxyphenylpyruvate concentrations in chloroplast subfractions. For further details see Materials and methods

\begin{tabular}{lcrr}
\hline $\begin{array}{l}\text { 4-Hydroxyphenyl- } \\
\text { pyruvate } \\
\text { applied }(\mu \mathrm{M})\end{array}$ & $\begin{array}{l}\text { Homogentisate formed } \\
\text { (pkat kg-1} \text { protein) }\end{array}$ & \\
\cline { 2 - 4 } & Envelope & Stroma & Thylakoids \\
\hline 1.5 & 5,170 & 306 & 111 \\
15.0 & 10,670 & 1,470 & 670 \\
150.0 & 158,900 & 44,170 & 8,610 \\
\hline
\end{tabular}

Rate of homogentisate formation in dependence on substrate concentration. 4-Hydroxyphenylpyruvate was applied in increasing concentrations $(1.5 ; 15$; $150 \mu \mathrm{M}$ ) to all three chloroplast subfractions. The highest conversion rate occurred at $150 \mu \mathrm{M}$ 4-hydroxyphenylpyruvate in the membranes as well as in the stroma fraction (Table 2).

Prenylquinol synthesis from 4-hydroxyphenylpyruvate by the envelope membrane. Envelope membranes are able to catalyze the enzymatic prenylation of homogentisate with phytyldiphosphate to give 2-methyl-6-phytylquinol (Soll et al. 1980) as the first intermediate in the $\alpha$-tocopherol synthesis. The incorporation of 4-hydroxyphenylpyruvate into 2-methyl-6-phytylquinol by envelope membranes could be shown according to the following two-step reaction:

4-hydroxyphenylpyruvate $+\mathrm{O}_{2}$

$\rightarrow$ homogentisate $+\mathrm{CO}_{2}$

homogentisate + phytyldiphosphate

$\rightarrow$ 2-methyl-6-phytylquinol $+\mathrm{CO}_{2}$

At two different substrate concentrations (30 and $70 \mu \mathrm{M} \quad$ 4-hydroxyphenylpyruvate) 36,1 and 156,4 pkat 2-methyl-6-phytylquinol per kg envelope protein were synthesized, respectively, linear with time.

\section{Discussion}

Homogentisate formation in chloroplasts was the "missing link" in connecting the shikimate pathway and the prenyl quinone synthesis. The plastidic shikimate pathway (Bickel et al. 1978) is thought to act in the stroma, whereas the synthesis of the prenylquinones $\alpha$-tocopherol and plastoquinone could exclusively be demonstrated in the membranes, especially the envelope (Soll et al. 1980). The homogentisate has to be transferred from the stroma to the membranes. 4-Hydroxyphenylpyruvate dioxygenase is present in the enve- lope in the highest specific activity, however, approx. $70 \%$ of the overall activity is found in the stroma. The dioxygenase might be at least a peripheral protein associated with the stromal face of the membranes.

It seems likely that homogentisate is formed directly from 4-hydroxyphenylpyruvate straight through the shikimate pathway. 4-Hydroxyphenylpyruvate formation from tyrosine by the L-amino acid oxidase of the thylakoids (Löffelhardt 1977) may be a bypass. The 4-hydroxyphenylpyruvate dioxygenase of peroxisomes studied earlier (Bickel et al. 1979) and reinvestigated here may play a role under semi-autotrophic conditions during the growth process (Bickel and Schultz 1974). In this case 4-hydroxyphenylpyruvate is supplied from tyrosine by a transaminase.

Financial support from the Deutsche Forschungsgemeinschaft is greatfully acknowledged.

\section{References}

Arnon, D.J. (1949) Copper enzymes in isolated chloroplasts. polyphenol oxidase in Beta vulgaris. Plant Physiol. 24, 1-15

Beevers, H. (1971) Comparative biochemistry of microbodies. In: Photosynthesis and photorespiration, pp. 483-493, Hatch, M.D., Osmond, C.B., Slayter, R.O., eds. Wiley Interscience, New York

Bickel, H., Schultz, G. (1974) Zum Phenolstoffwechsel während der Keimung von Gerste: Plastochinon- und FlavonoidSynthese aus Tyrosin des Endosperms. Ber. Dtsch. Bot. Ges. 87, 281-290

Bickel, H., Schultz, G. (1979) Shikimate pathway regulation in suspensions of intact spinach chloroplasts. Phytochemistry 18, 498-499

Bickel, H., Buchholz, B., Schultz, G. (1979) On the compartmentation of the biosynthesis of aromatic amino acids and prenylquinones in higher plants. In: Advances in the biochemistry and physiology of plant lipids, pp. 369-375, Appelqvist, L.A., Liljenberg, C., eds. Elsevier, Amsterdam

Bickel, H., Palme, L., Schultz, G. (1978) Incorporation of shikimate and other precursors into aromatic amino acids and prenylquinones of isolated spinach chloroplasts. Phytochemistry 17, 119-124

Buchholz, B., Reupke, B., Bickel, H., Schultz, G. (1979) Reconstitution of amino acid synthesis by combining spinach chloroplasts with other leaf organelles. Phytochemistry 18, 1109-1111

Buchholz, B., Schultz, G. (1980) Control of shikimate pathway in spinach chloroplasts by exogenous substrates. Z. Pflanzenphysiol. 100, 209-215

Douce, R., Holtz, R.B., Benson, A.A. (1973) Isolation and properties of the envelope of spinach chloroplasts. J. Biol. Chem. 248, 7215-7222

Douce, R., Joyard, J. (1977) Le chloroplaste. La Recherche 79, 527-537

Douce, R., Joyard, J. (1979) Isolation and properties of the envelope of spinach chloroplasts. In: Plant organelles, pp. 47-59, Reid, E., ed. Ellis Horwood, Chinchester

Douce, R., Joyard, J. (1979) Structure and function of the chloroplast envelope. Adv. Bot. Res. 7, 1-116 
Goodwin, T.W. (1965) Regulation of terpenoid synthesis in higher plants. In: Biosynthetic pathways in higher plants, pp. 57-71, Pridham, J.B., Swain, S., eds. Academic Press, London, New York

Hass, R., Siebertz, H.P., Wrage, K., Heinz, E. (1980) Localization of sulfolipid labeling within cells and chloroplasts. Planta 148, 238-244

Hutson, K.G., Threlfall, D.R. (1980) Synthesis of plastoquinone- 9 and phytylplastoquinone from homogentisate in lettuce chloroplasts. Biochim. Biophys. Acta 632, 630-648

Lindblad, B. (1971) Radiochemical assays for p-hydroxyphenyl pyruvate hydroxylase activity in human liver. Clin. Chim. Acta 34, 113-121

Löffelhardt, W. (1977) The biosynthesis of phenylacetic acids in the blue-green alga Anacytis nidulans: evidence for the involvement of a thylakoid-bound L-amino acid oxidase. Z. Naturforsch. 32 c, 345-350

Löffelhardt, W., Kindl, H. (1979) Conversion of 4-hydroxyphenylpyruvic acid into homogentisic acid at the thylakoid membrane of Lemna gibba. FEBS Lett 104, 332-334

Lowry, O.H., Rosenbrogh, N.J., Farr, A.L., Randall, R.J.

(1951) Protein mensurement with the Folin phenol reagent. J. Biol. Chem. 193, 265-275

McNeil, P.H., Foyer, C.H., Walker, D.A. (1981) Similarity of ribulose-1,5-bisphosphate carboxylase of isogenic diploid and tetraploid ryegrass (Lolium perenne L.) cultivars. Plant Physiol. 67, 530-534

Nakatani, H.Y., Barber, J. (1977) An improved method for isolating chloroplasts retaining their outer membranes. Biochim. Biophys. Acta 461, 510-512

Soll, J., Kemmerling, M., Schultz, G. (1980) Tocopherol and plastoquinone synthesis in spinach chloroplasts subfractions. Arch. Biochem. Biophys. 204, 544-550

Tolbert, N.E. (1971) Isolation of leaf peroxisomes. Methods Enzymol. 23, 665-687

Tolbert, N.E., Yamazaki, R.K., Oeser, A. (1970) Localization and properties of hydroxypyruvate and glyoxylate reductase in spinach leaf particles. J. Biol. Chem. 245, 5129-5136

Whistance, G.R., Threlfall, D.R. (1970) Biosynthesis of phytoquinones. Biochem. J. 117, 593-600

Received 8 March; accepted 26 May 1982 